Full paper

\title{
Nanocrystal light-emitting diodes based on type II nanoplatelets
}

\author{
Baiquan Liu $^{\mathrm{a}, 1}$, Savas Delikanli ${ }^{\mathrm{a}, 1}$, Yuan Gao ${ }^{\mathrm{a}}$, Didem Dede ${ }^{\mathrm{b}}$, Kivanc Gungor ${ }^{\mathrm{b}}$, \\ Hilmi Volkan Demir ${ }^{\mathrm{a}, \mathrm{b}, *}$ \\ ${ }^{\text {a }}$ Luminous! Center of Excellence for Semiconductor Lighting and Displays, School of Electrical and Electronic Engineering and School of Physical and Mathematical \\ Sciences, Nanyang Technological University, Singapore 639798, Singapore \\ ${ }^{b}$ Department of Electrical and Electronics Engineering, Department of Physics, UNAM-Institute of Materials Science and Nanotechnology, Bilkent University, Ankara \\ 06800, Turkey
}

\section{A R T I C L E I N F O}

\section{Keywords:}

Nanocrystal

Nanoplatelet

Light-emitting diode

Type II

Efficiency

\begin{abstract}
A B S T R A C T
Colloidal semiconductor nanoplatelets (NPLs) have recently emerged as a new family of semiconductor nanocrystals with distinctive structural and electronic properties originating from their atomically flat architecture. To date, type II NPLs have been demonstrated to possess great potential to optoelectronic applications, such as solar cells and lasers. Herein, nanocrystal light-emitting diodes (LEDs) based on type II NPLs have been developed. The photoluminescence quantum yield of these used type II NPL (CdSe/CdSe ${ }_{0.8} \mathrm{Te}_{0.2}$ core/crown) is close to $85 \%$. By exploring an effective inverted structure with the dual hole transport layer, the NPL-LEDs exhibit i) a turn-on voltage of $1.9 \mathrm{~V}$, ii) a maximum luminance of $34520 \mathrm{~cd} \mathrm{~m}^{-2}$, iii) an EQE of $3.57 \%$ and a PE of $9.44 \mathrm{~lm} \mathrm{~W}^{-1}$. Compared with previous NPL-based LEDs, the performance of our devices is remarkably enhanced. For example, the luminance is 350-fold higher than the best inverted NPL-based LED. The findings may not only represent a significant step for NPL-based LEDs, but also unlock a new opportunity that this class of type II NPLs materials are promising for developing high-performance LEDs.
\end{abstract}

\section{1. . Introduction}

Colloidal semiconductor nanocrystal light-emitting diodes (LEDs) have been actively studied for utilization in displays and lighting because they exhibit many exceptional properties including high efficiency, good color purity, low power consumption, light weight, long lifetime and flexibility [1-3]. Since the first demonstration of colloidal quantum-dot LED (QD-LED) [4], a large number of efforts have been made to enhance the device performance via the optimization of both material syntheses and device architectures. [5-7] As an impressive consequence, the external quantum efficiency (EQE) of QD-LEDs has reached $20.5 \%$ by inserting an insulating layer improving the device structure, which is comparable to that of state-of-the-art organic LEDs (OLEDs) [8].

Semiconductor nanoplatelets (NPLs), also known as colloidal quantum wells, have been introduced as a new class of solution-processed two-dimensional (2D) nanocrystals in recent years [9-12]. Aside from the core-only NPLs having different vertical thicknesses and tuned chemical compositions, various NPLs with heteroarchitectures (e.g., core/shell, core/crown, core/crown/shell) have also been synthesized to engineer the electronic structure and optical properties [13-15]. Thanks to the tight quantum confinement only in the vertical direction, NPLs possess many unique thickness-dependent optical characteristics, such as narrow emission band, ultrashort radiative fluorescence lifetime, giant oscillator strength transition and extremely large linear/ nonlinear absorption cross-sections [16-18]. Therefore, NPLs are highly attractive for optoelectronic applications including solar cells, lasers and LEDs [19-21]. However, compared to other types of semiconductor nanocrystal LEDs (e.g., those exploiting QDs, nanorods, and dot-inrods), the performance of NPL-based LEDs lags far behind. For example, Chen et al. designed a red NPL-based LED by using the core/shell CdSe/ CdZnS NPLs, achieving a maximum luminance of $4499 \mathrm{~cd} \mathrm{~m}^{-2}$, an EQE of $0.63 \%$ and a turn-on voltage of $4.7 \mathrm{~V}$, which is the best red NPLbased LED thus far. [22] Fan et al. reported a green NPL-based LED by utilizing the $\mathrm{CdSe}_{1-\mathrm{x}} \mathrm{S}_{\mathrm{x}}$ NPLs, obtaining a maximum luminance of $\sim$ $90 \mathrm{~cd} \mathrm{~m}^{-2}$, which is the best NPL-based LED using inverted structure [23]. More recently, Ling et al. have achieved a green NPL-based LED by employing organometal halide perovskite $\mathrm{CH}_{3} \mathrm{NH}_{3} \mathrm{PbBr}_{3}$ NPLs, yielding a maximum power efficiency (PE) of $1.0 \mathrm{~lm} \mathrm{~W}^{-1}$, the best PE for NPL-based LED [24]. Therefore, the question is: is it possible to

\footnotetext{
* Corresponding author at: Department of Electrical and Electronics Engineering, Department of Physics, UNAM-Institute of Materials Science and Nanotechnology, Bilkent University, Ankara 06800, Turkey.

E-mail address: hvdemir@ntu.edu.sg (H.V. Demir).

${ }^{1} \mathrm{~B}$. Liu and S. Delikanli contributed equally to this work.
} 
further enhance the performance of NPL-based LEDs?

Depending upon the relative alignment of the conduction band (CB) and valence band (VB) positions of the core material and those of the crown or shell materials, these hetero-NPLs can behave as type I or type II heterostructures [25-27]. For type I NPLs, both holes and electrons wave functions are confined in the same region due to a wider band gap crown or shell barrier. For type II NPLs, the electrons and holes wave functions are staggered with respect to each other. Over the last few years, type I and core-only NPLs have been synthesized and widely investigated, while negligible attention has been paid to type II NPLs. In fact, the first type II NPLs were just synthesized only three years ago [28]. To date, type-II NPLs have been demonstrated to possess great potential in optoelectronic applications such as solar cells and lasers [29]. In the terms of LED applications, previous NPL-based LEDs have been constructed by using type I NPLs and core-only NPLs. Unlike type I and core-only structures, type II NPLs can possess higher emission quantum yield and reduced overlap between the absorption and emission spectrum [30], rendering that type II NPLs are potentially very promising and beneficial to LEDs. However, no type II NPL has been reported to develop LEDs, to the best of our knowledge.

In this paper, type II NPLs, for the first time, have been used to develop LEDs. The photoluminescence quantum yield (PLQY) of the employed type II NPL (CdSe/CdSe ${ }_{0.8} \mathrm{Te}_{0.2}$ core/crown) is close to $85 \%$, which is the highest for NPLs utilized among all NPL-based LEDs. By taking advantage of an effective inverted structure with the dual hole transport layer (HTL), the LED can exhibit a very low turn-on voltage of $1.9 \mathrm{~V}$, a maximum luminance of $34520 \mathrm{~cd} \mathrm{~m}^{-2}$, an EQE of $3.57 \%$ and a $\mathrm{PE}$ as high as $9.44 \mathrm{~lm} \mathrm{~W}^{-1}$. Compared with previous NPL-based LEDs, the performance of our device is remarkably enhanced. For example, the luminance is 350 times higher than the previous best inverted NPLbased LED.

\section{Results and discussion}

\subsection{Sample characterization}

Colloidal CdSe/CdSe ${ }_{0.8} \mathrm{Te}_{0.2}$ core/crown NPLs were synthesized following the reported procedures with slight modifications. [30] Further details of the syntheses can be found in the Supporting information. CdSe/CdSe ${ }_{0.8} \mathrm{Te}_{0.2}$ core/crown NPLs exhibit roughly a rectangular shape, as can be seen from the high-angle annular dark-field scanning transmission electron microscopy (HAADF-STEM) image shown in Fig. 1a. In average, these type II core/crown NPLs possess a rather uniform lateral size of $\sim 25 \mathrm{~nm}$ in length by $\sim 8 \mathrm{~nm}$ in width. The optical properties of these type II CdSe/CdSe $e_{0.8} \mathrm{Te}_{0.2}$ core/crown NPLs were characterized via ultraviolet-visible absorbance and PL spectroscopy. Fig. 1b depicts the absorption and steady-state PL characteristics of the NPLs in toluene at room temperature. Unlike the core-only and type I NPLs previously employed in LEDs showing a large overlap and an extremely small Stokes shift between the absorption and PL spectra [22-24], type II NPLs exhibit considerably small spectral overlap between the absorption and PL. This decreased overlap enhances the QY of the film by decreasing the reabsorption rate in this system. Therefore, type II NPLs are promising for LEDs with their superior QY in film. The sharp excitonic features at $\sim 511$ and $\sim 482 \mathrm{~nm}$ observed in the absorption spectrum of $\mathrm{CdSe} / \mathrm{CdSe}_{0.8} \mathrm{Te}_{0.2}$ core/crown NPLs are associated with the electron-heavy hole and electron-light hole transitions, respectively, in the CdSe cores. The broad absorption feature beyond $\sim$ $511 \mathrm{~nm}$ is associated with the $\mathrm{CdSe}_{0.8} \mathrm{Te}_{0.2}$ crown and this broadening can be explained by the fluctuation of the Te composition in the $\mathrm{CdSe}_{0.8} \mathrm{Te}_{0.2}$ crown [30]. The normalized PL spectrum of the type II $\mathrm{CdSe} / \mathrm{CdSe}_{0.8} \mathrm{Te}_{0.2}$ core/crown NPLs shows a red emission peak at $\sim$ $599 \mathrm{~nm}$. To demonstrate that our NPLs structure is really type-II, we performed energy-dispersive X-ray spectroscopy (EDX) measurements on a single NPL using STEM at the core and crown regions. The results of this measurements are given in Fig. S1 (Supporting information).
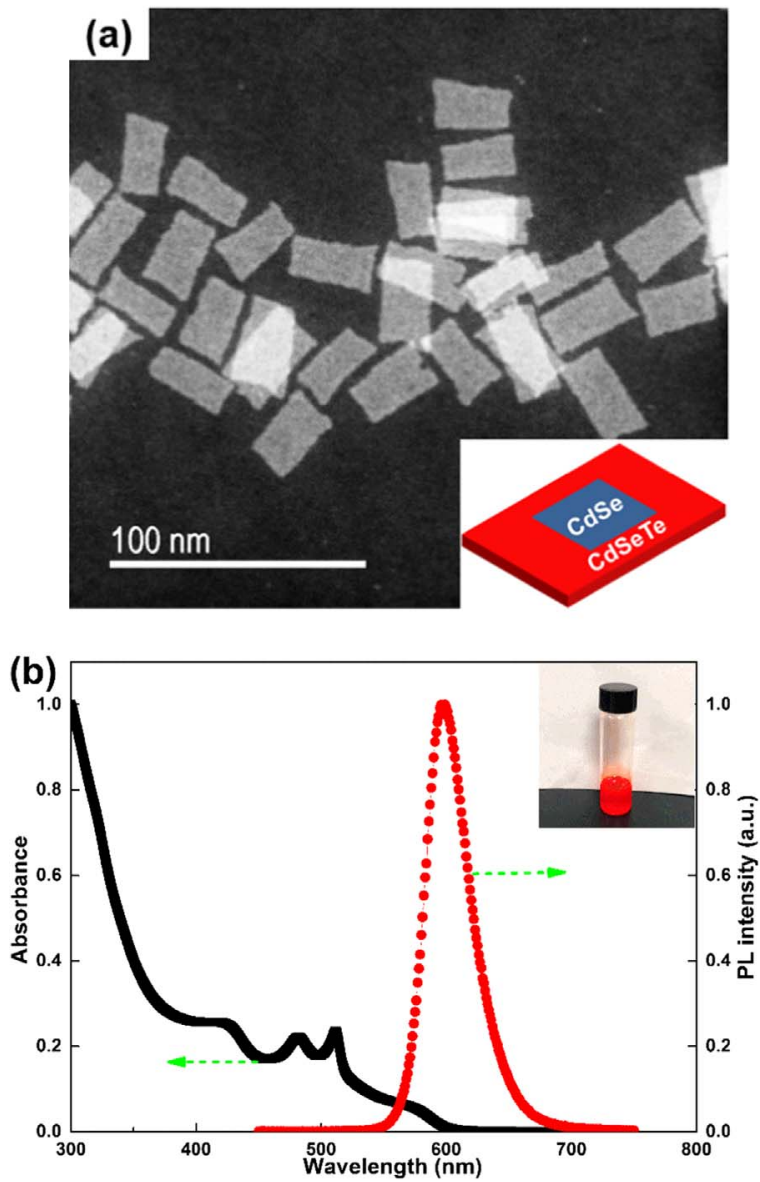

Fig. 1. (a) A HAADF-STEM image of the type II CdSe/CdSe ${ }_{0.8} \mathrm{Te}_{0.2}$ core/crown NPLs. Inset: a schematic structure of the NPLs. (b) Room-temperature absorbance and PL of NPLs in the toluene solution. Inset: a photograph of the NPLs solution.

These results show that there is only Se at the core while both Se and Te exist at the crown region, signifying the successful growth of CdSeTe crown layer on CdSe core. This is also in agreement with our optical measurements and previous reports. In addition, the excitonic features observed in the absorption spectrum and PL emission characteristics given in Fig. $1 \mathrm{~b}$ are in agreement with previously published results, [30] strongly indicating that these NPLs are type-II CdSe/CdSeTe NPLs.

\subsection{Device design strategy}

To achieve high-performance LEDs based on type II NPLs, here several strategies were adopted. First, an inverted structure was employed, which is advantageous for display applications, since the bottom transparent cathode of inverted LEDs can be directly connected to the low-cost n-type metal-oxide or amorphous-silicon thin film transistors [31]. In fact, high-performance inverted QD-LEDs have been realized in recent years. For example, inverted QD-LEDs can show a maximum $\mathrm{EQE}$ of $18 \%$, close to the theoretical maximum of $20 \%$ [32]. However, the best inverted NPL-based LED exhibits only a maximum luminance of $\sim 90 \mathrm{~cd} \mathrm{~m}^{-2}$. [23] To loosen the bottleneck, an urgent effort needs to be made to enhance the performance of inverted NPLbased LEDs.

Besides, a hybrid structure was exploited, where the NPL emitting layer (EML) is sandwiched between the inorganic zinc oxide ( $\mathrm{ZnO}$ ) electron transport layer (ETL) and organic HTL. Such a hybrid structure has the potential to achieve the high performance since i) various organic small molecules with high hole mobility can be selected as the effective HTLs and ii) HTLs can be deposited by thermal evaporation technique without any damage to the underlying layer, avoiding the 
solvent penetrating problem [32].

Then, unlike previously developed NPL-based LEDs that utilized the exchange of long chain ligands with the shorter ones, [22] a recyclable treatment on the type II CdSe/CdSe ${ }_{0.8} \mathrm{Te}_{0.2}$ core/crown NPLs was applied using hexane/ethyl acetate mixed solvent to control the surface ligand density here. Surface ligands are important tools for achieving successful surface passivation, elimination of surface defects, providing ink stability, and high PLQY. However, excessive ligands can form insulating layers since oleylamine and oleic acid organics utilized in the synthesis of the type II CdSe/CdSe ${ }_{0.8} \mathrm{Te}_{0.2}$ core/crown NPLs possess low electric conductivity, which in turn reduces the charge injection and transport inside the devices [33]. To accomplish the trade-off, the control of ligand density on NPL surfaces is used because it is more suitable than the surface ligand exchange to promote the performance of LEDs [34]. After the recyclable treatment, the PLQY of the new type II CdSe/CdSe ${ }_{0.8} \mathrm{Te}_{0.2}$ core/crown NPLs is close to $85 \%$, which is much higher than that of previous NPL-based LEDs (i.e., $20 \%$ for the best red NPL-based LEDs) [22]. The PLQY of $85 \%$ is also higher than that of previous works $[35,36]$. Besides, although type II NPLs have been reported, negligible attention has been paid on their surface ligand density. Since solvents play a vital role in controlling ligand density, [34] the presented recyclable treatment can be an effective approach to manage the ligand density of type II NPLs, which is beneficial to deeply understand type II NPLs and provides an exploration for the LED applications.

Finally, to boost the performance, the charge balance should be enhanced. In LEDs, the performance can be analyzed by EQE and PE. The EQE is defined as follows: [37]

$\mathrm{EQE}=\eta_{\text {out }} \cdot r \cdot q \cdot \gamma$

where $\eta_{\text {out }}$ is the outcoupling factor, $r$ is the fraction of excitons that can potentially radiatively decay, $q$ is the PLQY of emitters, and $\gamma$ is the charge balance $(\gamma \leq 1)$. The internal operation of the device usually does not influence $\eta_{\text {out }}$ and $\eta_{\text {out }}$ is about 0.2 according to the classical ray model. [35] Additionally, the terms $r$ and $q$ will be mostly set with the emitters. Hence, from the perspective of device engineering, the EQE is mostly sensitive to $\gamma$. Assuming the emission pattern is the Lambertian type, the connection between EQE and PE can be expressed as: [38]

$\mathrm{PE} \propto \frac{\mathrm{EQE}}{U}$

where $U$ is the driving voltage. Simply, PE can be considered as:

$\mathrm{PE} \propto \frac{\gamma}{U}$

Therefore, to achieve high PE, $\gamma$ should be high while the $U$ should be low. In other words, charges should be not only well balanced but also effectively transported. However, because of the unfavorable energy barriers between different layers, together with the fact that holes are minor charges in inorganic materials, charges balance and transport are usually unsatisfactory [8]. To address these issues in NPL-based LEDs, the exploration of ideal HTL is one of the most effective ways, yet virtually no work has been reported in this direction. Here, we first used 4,4'-bis-( $m$-tolyphenylamino)biphenyl (TPD), 4,4',4"'-Tri( $N$-carbazolyl) triphenylamine (TCTA) and 4,4-N,N-dicarbazolebiphenyl (CBP) as the single HTL to investigate the performance of NPL-based LEDs. Furthermore, we explored a dual-HTL to enhance the performance.

Taking the above factors into account, high-performance LEDs based on type II NPLs may be expected. As shown in Fig. 2, the structure of LEDs with single HTL is indium tin oxide (ITO)/ ZnO/ type II NPL/ HTLs $(60 \mathrm{~nm}) / \mathrm{MoO}_{3}(10 \mathrm{~nm}) / \mathrm{Al}(100 \mathrm{~nm})$, where ITO is the cathode, $\mathrm{ZnO}$ is the ETL, NPL is CdSe/CdSe ${ }_{0.8} \mathrm{Te}_{0.2}(6 \mathrm{mg} / \mathrm{mL}, 2000 \mathrm{rpm}, 45 \mathrm{~s})$, $\mathrm{MoO}_{3}$ is the hole injection layer (HIL), Al is the anode, and HTLs are TPD (device R1), TCTA (device R2) and CBP (device R3).

\subsection{Device with single HTL}

As shown in Fig. 3a and Table 1, Device R3 shows the lowest voltage and the highest luminance among all LEDs with a single HTL, suggesting that CBP is the best single HTL. For example, the turn-on voltage (the voltage when the luminance can be detected, $\geq 0.01 \mathrm{~cd} \mathrm{~m}^{-2}$ ) of Device R3 is $2.2 \mathrm{~V}$, which is lower than those of Devices R1 (2.6 V) and R2 $(3.0 \mathrm{~V})$. At $1000 \mathrm{~cd} \mathrm{~m}^{-2}$, the voltage of Device R3 is $4.3 \mathrm{~V}$, which is also lower than those of Devices R1 (4.6 V) and R2 (5.1 V). On the other hand, the maximum luminance of Device R3 is $22920 \mathrm{~cd} \mathrm{~m}^{-2}$, which is significantly higher than those of Devices $\mathrm{R} 1\left(8228 \mathrm{~cd} \mathrm{~m}^{-2}\right)$ and R2 (14350 $\left.\mathrm{cd} \mathrm{m}^{-2}\right)$.

The phenomenon that facilitates Device R3 to exhibit the best performance among LEDs with a single HTL can be explained as follows. Since all layers in these LEDs are similar except the HTL, the low voltages and high luminances can be attributed to the selection of CBP. For HTLs, hole mobility, hole injection efficiency, hole-blocking ability, and energy gap play critical roles in LEDs. [43,44] For Device R1, although TPD has the highest hole mobility among these single HTLs $\left(1.4 \times 10^{-3} \mathrm{~cm}^{2} \mathrm{~V}^{-1} \mathrm{~s}^{-1}\right)$ and strong electron-blocking ability due to its LUMO of $2.4 \mathrm{eV}$, the HOMO of TPD is not deep enough (5.4 eV) [41]. As a result, there is a large energy barrier between the NPL and TPD $(\sim 0.6 \mathrm{eV})$ (Fig. 2c), which is unfavorable for the hole transport, leading to a large number of holes accumulated at the EML/ TPD interface. Such hole accumulation can easily result in NPL charging, which is detrimental to the device performance since such charging can lead to the NPL luminescence quenching via the nonradiative Auger recombination mechanism. [45] Hence, although the very high hole mobility and narrow energy gap of TPD render that device R1 exhibits a high current density, [46] Device R1 shows unsatisfactory luminance and poor efficiency due to the Auger recombination (Fig. 3b). For Device R2, because the hole mobility of TCTA is relatively low $\left(8.0 \times 10^{-5} \mathrm{~cm}^{2} \mathrm{~V}^{-1} \mathrm{~s}^{-1}\right)$, the hole transport ability is poor for the TCTA material, giving rise to the high voltage, although TCTA has a stronger electron-blocking ability than CBP because the lowest unoccupied molecular orbital (LUMO) of TCTA $(2.3 \mathrm{eV})$ is higher than that of CBP $(2.5 \mathrm{eV})[41,42]$. For Device R3, since CBP possesses a high hole mobility $\left(10^{-3} \mathrm{~cm}^{2} \mathrm{~V}^{-1} \mathrm{~s}^{-1}\right)$, holes can be effectively transported. [47] In addition, the highest occupied molecular orbital (HOMO) of CBP is $5.9 \mathrm{eV}$, which is almost the same as that of the NPL, indicating that holes can be easily transported from CBP to the NPL [42]. Therefore, CBP is the most suitable single HTL to enhance the charge balance and transport, allowing for the low voltage.

According to Eqs. (1) and (3), because of the well balanced and effectively transported charges in Device R3, both the EQE and PE can be enhanced. As shown in Fig. $3 \mathbf{b}$ and Table 1, the efficiency of Device R3 is higher than those of Devices R1 and R2. For instance, the maximum EQE of Device R3 is 2.01\%, higher than those of R1 $(0.49 \%)$ and R2 (1.07\%). At $1000 \mathrm{~cd} \mathrm{~m}^{-2}$, the EQE of Device R3 is $1.41 \%$, higher than Devices R1 (0.43\%) and R2 (1.02\%). Also, the maximum PE of R3 is $4.64 \mathrm{~lm} \mathrm{~W}^{-1}$, again higher than $\mathrm{R} 1 \quad\left(0.75 \mathrm{~lm} \mathrm{~W}^{-1}\right)$ and $\mathrm{R} 2$ $\left(1.49 \mathrm{~lm} \mathrm{~W}^{-1}\right)$. At $1000 \mathrm{~cd} \mathrm{~m}^{-2}$, the PE of Device $\mathrm{R} 3$ is $2.4 \mathrm{~lm} \mathrm{~W} \mathrm{~m}^{-1}$, similarly higher than $\mathrm{R} 1\left(0.66 \mathrm{~lm} \mathrm{~W}^{-1}\right)$ and $\mathrm{R} 2\left(1.48 \mathrm{~lm} \mathrm{~W}^{-1}\right)$.

\subsection{Device with dual-HTLs}

In OLEDs, multilayer structures have been usually employed (i.e., $\geq 3$ electron injection layers, $\mathrm{p}$-doping/n-doping layers), which are used to effectively enhance the charge injection and balance. [48-50] However, multilayer structures are difficult to be introduced in colloidal semiconductor nanocrystal LEDs due to the solution-processed technique, limiting the device performance [37]. Hence, although the structures of previous NPL-based LEDs are simple (i.e., using a single HTL and a single ETL), the charge injection and balance may be not optimized. Galvanized by the fact that the performance of NPL-based LEDs can be greatly affected by different HTLs, we further improved the 


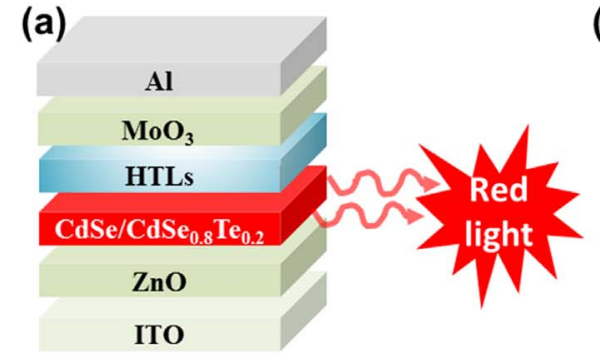

(c)

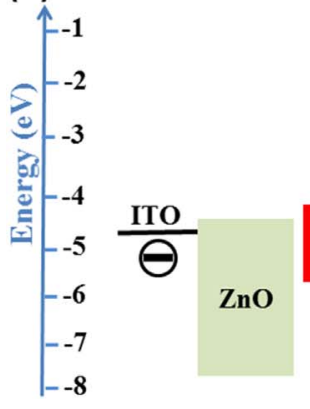

(b)

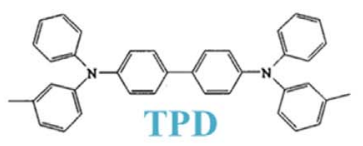
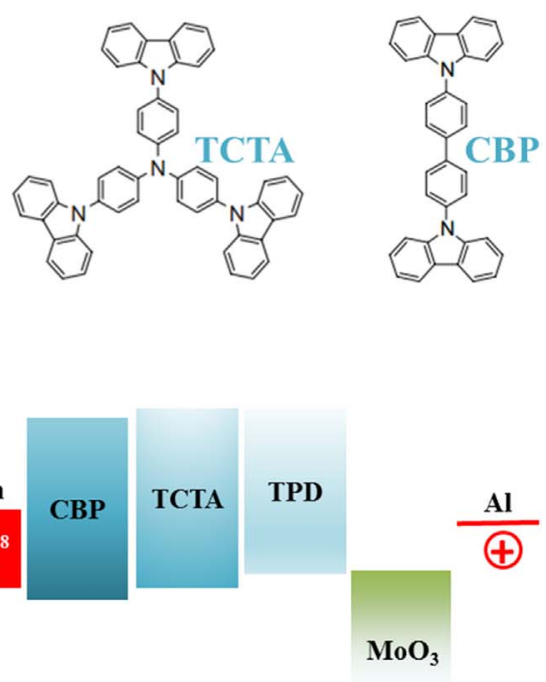

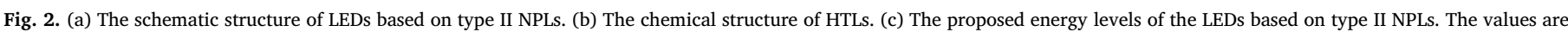
taken from the literature. $[8,22,39-42]$.
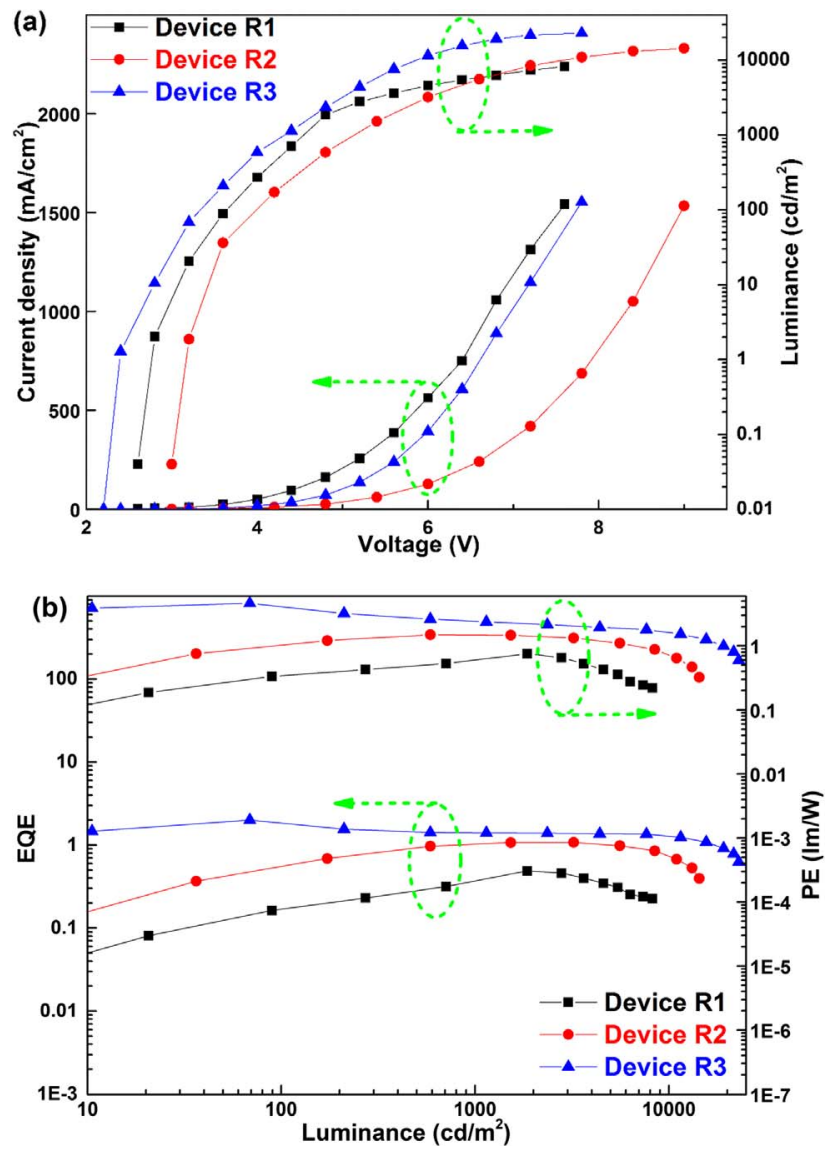

Fig. 3. (a) Current density and luminance as a function of the voltage across Devices R1, R2 and R3. (b) EQE and PE as a function of the luminance for Devices R1, R2 and R3.

device structure by adopting a dual-HTL architecture. Since the HOMO of TCTA is appropriately located between those of NPLs and TPD, we developed a LED with the dual-HTL TCTA $(10 \mathrm{~nm}) /$ TPD $(50 \mathrm{~nm})$ (Devices R4), while other layers are similar to those of the LED with a
Table 1

Summary of the performance levels of LEDs.

\begin{tabular}{llllll}
\hline Device & $\begin{array}{l}\mathrm{V}_{\text {on } / 1000}{ }^{\mathrm{a}} \\
(\mathrm{V})\end{array}$ & $\begin{array}{l}\mathrm{EQE}_{\max / 1000}{ }^{\mathrm{b}} \\
(\%)\end{array}$ & $\begin{array}{l}\mathrm{PE}_{\max / 1000}{ }^{\mathrm{c}} \\
\left(\mathrm{lm} \mathrm{W}^{-1}\right)\end{array}$ & $\begin{array}{l}\mathrm{L}_{\max }{ }^{\mathrm{d}} \\
\left(\mathrm{cd} \mathrm{m}^{-2}\right)\end{array}$ & $\mathrm{CIE}^{\mathrm{e}}$ \\
\hline $\mathrm{R} 1$ & $2.6 / 4.6$ & $0.49 / 0.43$ & $0.75 / 0.66$ & 8228 & $(0.61,0.38)$ \\
$\mathrm{R} 2$ & $3.0 / 5.1$ & $1.07 / 1.02$ & $1.49 / 1.48$ & 14350 & $(0.61,0.38)$ \\
$\mathrm{R} 3$ & $2.2 / 4.3$ & $2.01 / 1.41$ & $4.64 / 2.40$ & 22920 & $(0.61,0.38)$ \\
$\mathrm{R} 4$ & $1.9 / 3.4$ & $3.57 / 3.20$ & $9.44 / 7.10$ & 34520 & $(0.61,0.38)$ \\
\hline
\end{tabular}

${ }^{\text {a }}$ Turn-on voltage and the voltage at $1000 \mathrm{~cd} \mathrm{~m}^{-2}$.

${ }^{\mathrm{b}}$ Maximum EQE and $\mathrm{EQE}$ at $1000 \mathrm{~cd} \mathrm{~m}^{-2}$.

c Maximum PE and PE at $1000 \mathrm{~cd} \mathrm{~m}^{-2}$.

d Maximum luminance.

e The Commission International de I'Eclairage (CIE) coordinates at $1000 \mathrm{~cd} \mathrm{~m}^{-2}$.

single HTL. The cross-sectional scanning electron microscope (SEM) image of Device R4 is depicted in Fig. S2 (Supporting information).

As shown in Fig. 4a and Table 1, Device R4 shows low voltage and high luminance. For example, the turn-on voltage of Device R4 is $1.9 \mathrm{~V}$, which is the lowest among NPL-based LEDs. The sub-bandgap voltage is also observed by other groups, which can be attributed to the efficient charge injection at low bias $[8,32]$. At $1000 \mathrm{~cd} \mathrm{~m}^{-2}$, the voltage is still as low as $3.4 \mathrm{~V}$. Remarkably, the maximum luminance of Device R4 is $34520 \mathrm{~cd} \mathrm{~m}^{-2}$, which is the highest for NPL-based LEDs. Due to the effective dual-HTL, the efficiency of device R4 is also significantly enhanced. For example, Device R4 can exhibit a maximum EQE of 3.57\% and a maximum PE of $9.44 \mathrm{~lm} \mathrm{~W}^{-1}$, which is the highest for NPL-based LEDs. To the best of our knowledge, this device outperforms previous NPL-based LEDs [22-24]. For example, the luminance of Device R4 with the inverted structure is 350 -fold larger than that of the best inverted NPL-based LED [23].

Fig. 5 depicts the electroluminance (EL) properties of Device R4. Unlike previous NPL-based LEDs of which the EL spectra are obviously redshifted compared with their PL spectra here, the EL spectrum $(599 \mathrm{~nm})$ almost coincides with the PL spectrum $(599 \mathrm{~nm})$ for Device R4 (Fig. 5a). Such well complete overlap between the EL and PL spectrum demonstrates the efficient recombination of holes and electrons on NPLs in the LEDs. [51] Besides, the EL spectra are very stable at different luminance levels, as shown in Fig. $5 \mathbf{b}$. In addition, the color 

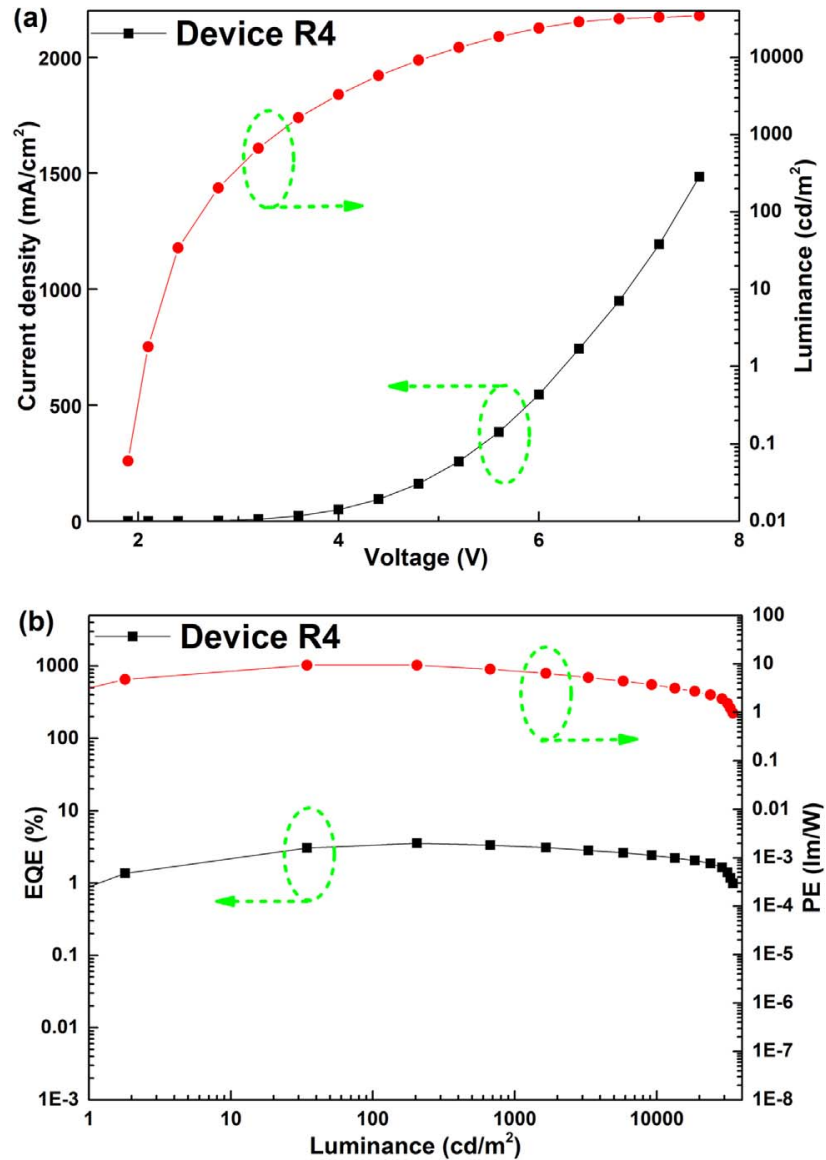

Fig. 4. (a) Current density and luminance of device R4. (b) EQE and PE of device R4.

of Device R4 is well located in the red region with the CIE coordinates of $(0.61,0.38)$, as shown in Fig. $5 \mathbf{c}$ and $\mathbf{d}$.

\subsection{Insights of the effect of the dual-HTL}

Upon using the dual-HTL TCTA/TPD, the performance of Device R4 was made significantly higher than that of the device with the best single HTL (Device R3). Hence, to understand the origin of the high performance of Device R4, the effect of the dual-HTL TCTA/TPD needs to be unveiled. Particularly, the roles of the dual-HTL and the best single HTL CBP in the device performance will need to be comprehensively discussed.

First, it is noted the electron mobility of CBP is as high as $10^{-4} \mathrm{~cm}^{2}$ $\mathrm{V}^{-1} \mathrm{~s}^{-1}$, [47] indicating that electrons can escape from the EML when they obtain enough energy at high voltages/ luminances, although there is a LUMO barrier between the CBP and the NPL. Since the electron leakage is detrimental to the efficiency, [43] CBP is not so ideal to be the HTL. On the other hand, TCTA is a p-type material and its electron mobility can be negligible, [41] implying that electrons can be well confined in the EML. Additionally, the LUMO of TCTA is higher than that of CBP, indicating that TCTA possesses a better electronblocking ability, which can further reduce the electron leakage. As a result, more electrons can meet more holes to form more excitons in Device R4 than Device R3, leading to higher performance.

Besides, the hole injection efficiency of HTLs is essential for high performance. [43] To determine the hole injection efficiency of CBP and TCTA/TPD, hole-only devices have been fabricated. [46] The structures are ITO/ poly(3,4-ethylenedioxythiophene): polystyrene sulfonate (PEDOT: PSS, $40 \mathrm{~nm}) / \mathrm{CdSe} / \mathrm{CdSe}_{0.8} \mathrm{Te}_{0.2}(6 \mathrm{mg} / \mathrm{mL}$, $2000 \mathrm{rpm}, 45 \mathrm{~s}) / \mathrm{CBP}$ or TCTA/TPD $(60 \mathrm{~nm}) / \mathrm{MoO}_{3}(5 \mathrm{~nm}) / \mathrm{Al}$ $(100 \mathrm{~nm})$, where PEDOT: PSS is a well-known p-type material and functions as the electron blocking layer for the hole-only devices [6]. As shown in Fig. 6, the TCTA/TPD based device shows higher current density than the CBP based device, indicating that the dual-HTL TCTA/ TPD possesses a higher hole injection efficiency than the single HTL CBP. Hence, holes are more easily injected from the TCTA/TPD dualHTL to the EML. The reason why TCTA/TPD possesses a higher hole injection efficiency can be explained as follows. On one hand, when inserting a thin $\mathrm{MoO}_{3}$ between ITO and TPD, an Ohmic hole injection can be easily formed, [52] implying that holes are barrier-free transported to the dual-HTL. However, despite the fact that $\mathrm{MoO}_{3}$ can enhance the hole injection of ITO/ CBP interface, it is not as efficient as that of the ITO/ TPD interface [53]. On the other hand, since the HOMO of TCTA $(5.7 \mathrm{eV})$ is well located between TPD $(5.4 \mathrm{eV})$ and NPLs ( $6.0 \mathrm{eV}$ ), a stepwise structure is formed, which is favourable to the hole transport [43]. Although the hole mobility of TCTA is not high, the reduced energy barrier resulted from the dual-HTL can vastly enhance the hole transport since the hole mobility has less influence on the performance compared with the energy level alignment [54-56]. Hence, holes can be easily injected into the EML via the stepwise structure. In other words, more holes can be injected into the EML in Device R4 than Device R3, which is key to the high performance.

Considering the high electron mobility of $\mathrm{ZnO}\left(1.3 \times 10^{-3} \mathrm{~cm}^{2} \mathrm{~V}^{-1}\right.$ $\mathrm{s}^{-1}$ ) and small LUMO barrier between $\mathrm{ZnO}$ and NPLs, [57] electrons are very easily injected into the EML from the cathode. Hence, the number of electrons can surpass that of holes, leading to a poor charge balance. In fact, when using $\mathrm{ZnO}$ as the ETL, electrons are usually the major carriers in colloidal semiconductor nanocrystal LEDs, regardless of inverted or normal structures [37]. Previously, due to the strong electron ability of $\mathrm{ZnO}$, Dai et al. used an insulating layer $6 \mathrm{~nm}$ poly(methylmethacrylate) to limit the electron transporting from $\mathrm{ZnO}$ into the EML, which is expected to balance the number of electrons and holes. [8] Here, to achieve the charge balance, we adopt an alternative way by increasing the hole injection efficiency from the dual-HTL TCTA/TPD instead of decreasing the electron injection efficiency. Therefore, due to the better charge balance, it is reasonable that Device R4 outperforms Device R3 according to Eqs. (1) and (2).

Furthermore, charging can degrade the emitting properties of NPLs, regardless of the electroluminescence or photoluminescence [8]. Due to the different workfunction, a spontaneous charge transfer process can occur upon NPLs directly contacting the charge transport layer, resulting in charged NPLs, which causes inefficient trion emissions [58,59]. As shown in Fig. 7, the average photoluminescence lifetime of the NPL film is $6.5 \mathrm{~ns}$, which is decreased to $0.72 \mathrm{~ns}$ after depositing the top CBP film while remained $2.39 \mathrm{~ns}$ after depositing the TCTA/TPD film. Therefore, the dual-HTL TCTA/TPD is beneficial to maintain charge neutrality of NPL emitters and preserve their superior emitting properties, which is another factor that contributes to the high performance of Device R4.

\section{Conclusions}

In summary, LEDs based on type II NPLs have been successfully developed. By incorporating the highly efficient $\mathrm{CdSe} / \mathrm{CdSe}_{0.8} \mathrm{Te}_{0.2}$ core/crown type II NPLs in effective inverted architecture with a dual HTL, low voltage, high luminance and high efficiency has been simultaneously achieved. This NPL-LED exhibits a turn-on voltage of $1.9 \mathrm{~V}$, a maximum luminance of $34520 \mathrm{~cd} \mathrm{~m}^{-2}$, a high EQE of $3.57 \%$ and a high $\mathrm{PE}$ of $9.44 \mathrm{~lm} \mathrm{~W}^{-1}$, which are remarkably superior to the reports of previous NPL-based LEDs (i.e., 2 orders of magnitude higher than the previous best inverted NPL-based LED). The findings may not only represent a significant step for the NPL-based LEDs, but unlock a new opportunity that this class of materials (type II NPLs) are promising for developing high-performance LEDs. 

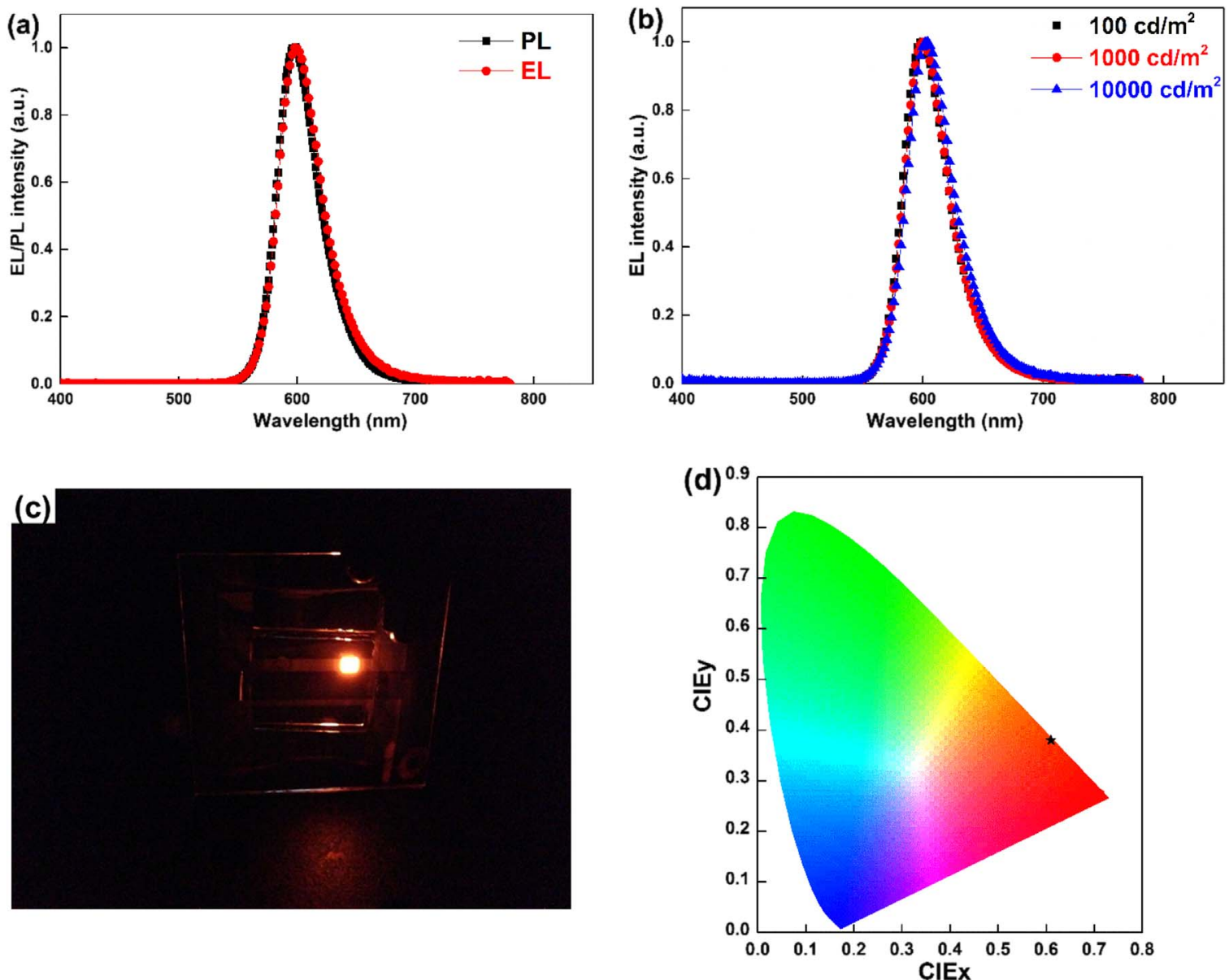

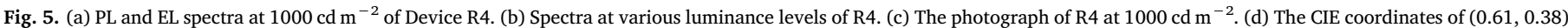
at $1000 \mathrm{~cd} \mathrm{~m}^{-2}$.

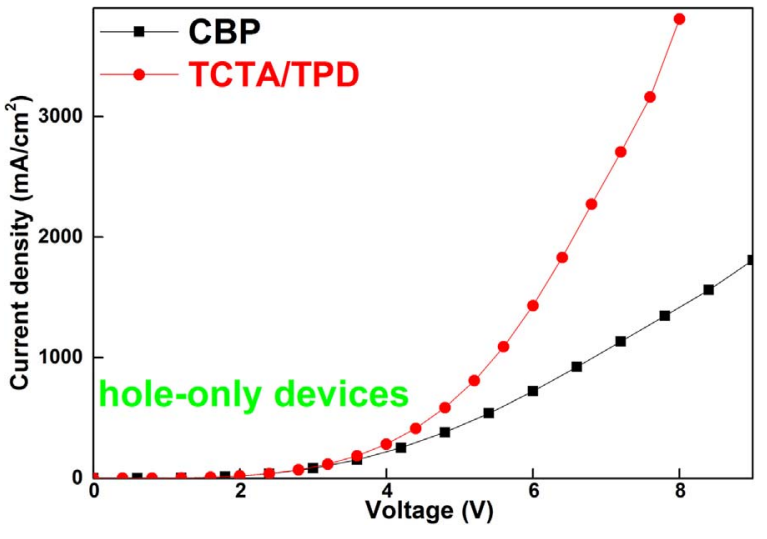

Fig. 6. Current density vs bias voltage in hole-only devices.

\section{Methods}

Without breaking the vacuum, all material layers were thermally deposited at a base pressure of $2.0 \times 10^{-4} \mathrm{~Pa}$. The thicknesses of all layers were controlled by the quartz crystal oscillators. After preparation under a nitrogen atmosphere using epoxy glue and glass slides, all devices were encapsulated immediately. The CIE coordinates and EL spectra were recorded via a PR650 Spectra Scan spectrometer. By utilizing a computer-controlled source meter, the current density-voltageluminance properties were measured simultaneously.

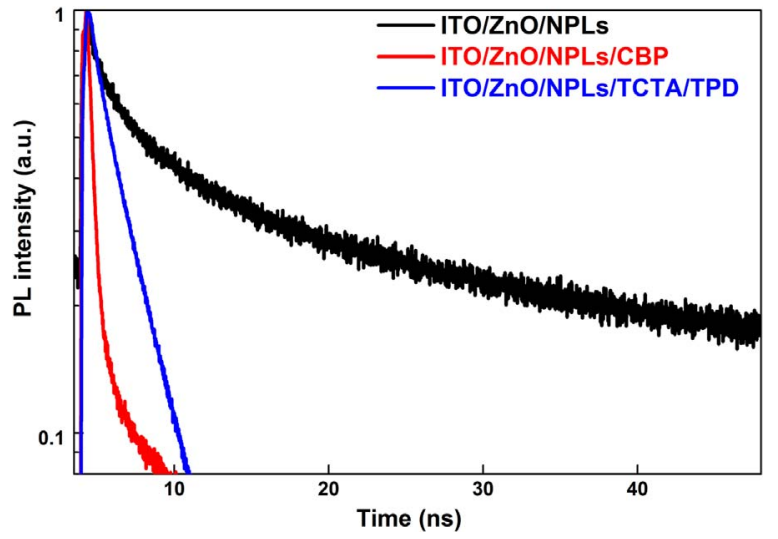

Fig. 7. Time-resolved photoluminescence decay of NPL films contacting different layers. The thicknesses of layers are the same as those in LEDs.

\section{Acknowledgements}

This research is supported by the National Research Foundation, Prime Minister's Office, Singapore under its Investigatorship program (NRF-NRFI2016-08) and the Singapore Agency for Science, Technology and Research (A*STAR) SERC Pharos Program under Grant No. 15273 00025. HVD gratefully acknowledges TUBA-GEBIP. The electron microscopy imaging was performed at the Facility for Analysis, Characterization, Testing and Simulation (FACTS) at Nanyang Technological University, Singapore. 
Notes

The authors declare no competing financial interest.

\section{Appendix A. Supporting information}

Supplementary data associated with this article can be found in the online version at http://dx.doi.org/10.1016/j.nanoen.2018.02.004.

\section{References}

[1] D.V. Talapin, J. -S. Lee, M.V. Kovalenko, E.V. Shevchenko, Prospects of colloidal nanocrystals for electronic and optoelectronic applications, Chem. Rev. 110 (2010) 389-458.

[2] S.D. Stranks, H.J. Snaith, Metal-halide perovskites for photovoltaic and lightemitting devices, Nat. Nanotechnol. 10 (2015) 391-402.

[3] L. Jing, S.V. Kershaw, Y. Li, X. Huang, Y. Li, A.L. Rogach, M. Gao, Aqueous based semiconductor nanocrystals, Chem. Rev. 116 (2016) 10623-10730.

[4] V.L. Colvin, M.C. Schlamp, A.P. Alivisatos, Light-emitting diodes made from cadmium selenide nanocrystals and a semiconducting polymer, Nature 370 (1994) 354-357.

[5] S. Coe, W.-K. Woo, M.G. Bawendi, V. Bulovic, Electro-luminescence from single monolayers of nanocrystals in molecular organic devices, Nature 420 (2002) 800-803.

[6] Q.J. Sun, Y.A. Wang, L.S. Li, D. Wang, T. Zhu, J. Xu, C. Yang, Y. Bright Li, Multicoloured light-emitting diodes based on quantum dots, Nat. Photonics (1) (2007) 717-722

[7] Y.X. Yang, Y. Zheng, W.R. Cao, A. Titov, J. Hyvonen, J.R. Manders, J.G. Xue, P.H. Holloway, L. Qian, High-efficiency light-emitting devices based on quantum dots with tailored nanostructures, Nat. Photonics 9 (2015) 259-266.

[8] X.L. Dai, Z.X. Zhang, Y.Z. Jin, Y. Niu, H.J. Cao, X.Y. Liang, L.W. Chen, J.P. Wang, X.G. Peng, Solution-processed, high performance light-emitting diodes based on quantum dots, Nature 515 (2014) 96-100.

[9] S. Ithurria, M.D. Tessier, B. Mahler, R.P.S.M. Lobo, B. Dubertret, A.L. Efros, Colloidal nanoplatelets with two dimensional electronic structure, Nat. Mater. 10 (2011) 936-941.

[10] J.Q. Grim, S. Christodoulou, F. Di Stasio, R. Krahne, R. Cingolani, L. Manna, I. Moreels, Continuous-wave biexciton lasing at room temperature using solutionprocessed quantum wells, Nat. Nanotechnol. 9 (2014) 891-895.

[11] C.E. Rowland, I. Fedin, H. Zhang, S.K. Gray, A.O. Govorov, D.V. Talapin, R.D. Schaller, Picosecond energy transfer and multiexciton transfer outpaces auger recombination in binary cdse nanoplatelet solids, Nat. Mater. 14 (2015) 484-489.

[12] A. Riedinger, F.D. Ott, A. Mule, S. Mazzotti, P.N. Knusel, S.J.P. Kress, F. Prins, S.C. Erwin, D.J. Norris, Anintrinsicgrowth instability inisotropic materials leads to quasi-two-dimensional nanoplatelets, Nat. Mater. (2017), http://dx.doi.org/10. 1038/nmat4889.

[13] B. Mahler, B. Nadal, C. Bouet, G. Patriarche, B. Dubertret, Core/shell colloida semiconductor nanoplatelets, J. Am. Chem. Soc. 134 (2012) 18591-18598.

[14] A. Prudnikau, A. Chuvilin, M. Artemyev, CdSe-CdS nanoheteroplatelets with efficient photoexcitation of central CdSe region through epitaxially grown CdS wings, J. Am. Chem. Soc. 135 (2013) 14476-14479.

[15] M.D. Tessier, P. Spinicelli, D. Dupont, G. Patriarche, S. Ithurria, B. Dubertret, Efficient exciton concentrators built from colloidal core/crown CdSe/CdS semiconductor nanoplatelets, Nano Lett. 14 (2014) 207-213.

[16] M.D. Tessier, C. Javaux, I. Maksimovic, V. Loriette, B. Dubertret, Spectroscopy of single CdSe nanoplatelets, ACS Nano 6 (2012) 6751-6758.

[17] K. Wu, Q. Li, Y. Jia, J.R. McBride, Z.-X. Xie, T. Lian, Efficient and ultrafast formation of long-lived charge-transfer exciton state in atomically thin cadmium selenide/ cadmium telluride Type-II heteronanosheets, ACS Nano 9 (2015) 961-968.

[18] Y. Kelestemur, B. Guzelturk, O. Erdem, M. Olutas, K. Gungor, H.V. Demir, Plateletin-box colloidal quantum wells: CdSe/CdS@CdS core/crown@shell heteronanoplatelets, Adv. Funct. Mater. 26 (2016) 3570-3579.

[19] L.T. Kunneman, J.M. Schins, S. Pedetti, H. Heuclin, F.C. Grozema, A.J. Houtepen, B. Dubertret, L.D.A. Siebbeles, Nature and decay pathways of photoexcited states in CdSe and CdSe/CdS nanoplatelets, Nano Lett. 14 (2014) 7039-7045.

[20] C. She, I. Fedin, D.S. Dolzhnikov, P.D. Dahlberg, G.S. Engel, R.D. Schaller, D.V. Talapin, Red, yellow, green, and blue amplified spontaneous emission and lasing using colloidal CdSe nanoplatelets, ACS Nano 9 (2015) 9475-9485.

[21] F.T. Rabouw, J.C. Bok, P. Spinicelli, B. Mahler, M. Nasilowski, S. Pedetti, B. Dubertret, D. Vanmaekelbergh, Temporary charge carrier separation dominates the photoluminescence decay dynamics of colloidal CdSe nanoplatelets, Nano Lett. 16 (2016) 2047-2053.

[22] Z. Chen, B. Nadal, B. Mahler, H. Aubin, B. Dubertret, Quasi-2D colloidal semiconductor nanoplatelets for narrow electroluminescence, Adv. Funct. Mater. 24 (2014) 295-302.

[23] F. Fan, P. Kanjanaboos, M. Saravanapavanantham, E. Beauregard, G. Ingram, E. Yassitepe, M.M. Adachi, O. Voznyy, A.K. Johnston, G. Walters, et al., Colloidal $\mathrm{CdSe}_{1-\mathrm{x}} \mathrm{S}_{\mathrm{X}}$ nanoplatelets with narrow and continuously-tunable electroluminescence, Nano Lett. 15 (2015) 4611-4615.

[24] Y. Ling, Z. Yuan, Y. Tian, X. Wang, J.C. Wang, Y. Xin, K. Hanson, B. Ma, H. Gao, Bright light-emitting diodes based on organometal halide perovskite nanoplatelets, Adv. Mater. 28 (2016) 305-311.
[25] M.D. Tessier, B. Mahler, B. Nadal, H. Heuclin, S. Pedetti, B. Dubertret, Spectroscopy of colloidal semiconductor core/shell nanoplatelets with high quantum yield, Nano Lett. 13 (2013) 3321-3328.

[26] C. She, I. Fedin, D.S. Dolzhnikov, A. Demortiere, R. D. Schaller, M. Pelton, D.V. Talapin, Low-threshold stimulated emission using colloidal quantum wells, Nano Lett. 14 (2014) 2772-2777.

[27] M. Olutas, B. Guzelturk, Y. Kelestemur, A. Yeltik, S. Delikanli, H.V. Demir, Lateral size-dependent spontaneous and stimulated emission properties in colloidal CdSe nanoplatelets, ACS Nano 9 (2015) 5041-5050.

[28] S. Pedetti, S. Ithurria, H. Heuclin, G. Patriarche, B. Dubertret, Type-II CdSe/CdTe core/crown semiconductor nanoplatelets, J. Am. Chem. Soc. 136 (2014) $16430-16438$.

[29] Q. Li, Z. Xu, J.R. McBride, T. Lian, Colloidal CdSe/CdTe core/crown type-II nanoplatelet heterostructures, ACS Nano 11 (2017) 2545-2553.

[30] Y. Kelestemur, B. Guzelturk, O. Erdem, M. Olutas, T. Erdem, C.F. Usanmaz, K. Gungor, H.V. Demir, CdSe/CdSe ${ }_{1-x} \mathrm{Te}_{\mathrm{x}}$ core/crown heteronanoplatelets: tuning the excitonic properties without changing the thickness, J. Phys. Chem. C. 121 (2017) 4650-4658.

[31] H. Zhang, H. Li, X. Sun, S. Chen, Inverted quantum-dot light-emitting diodes fabricated by all-solution processing, ACS Appl. Mater. Interfaces 8 (2016) 5493-5498.

[32] B.S. Mashford, M. Stevenson, Z. Popovic, C. Hamilton, Z. Zhou, C. Breen, J. Steckel, V. Bulovic, M. Bawendi, S. Coe-Sullivan, P.T. Kazlas, High-efficiency quantum-dot light-emitting devices with enhanced charge injection, Nat. Photonics 7 (2013) 407-412.

[33] J. -S. Lee, M.V. Kovalenko, J. Huang, D.S. Chung, D.V. Talapin, Band-like transport, high electron mobility and high photoconductivity in all-inorganic nanocrystal arrays, Nat. Nanotechnol. 6 (2011) 348-352.

[34] J. Li, L. Xu, T. Wang, J. Song, J. Chen, J. Xue, Y. Dong, B. Cai, Q. Shan, B. Han, H. Zeng, 50-fold EQE improvement up to $6.27 \%$ of solution-processed all-inorganic perovskite CsPbBr ${ }_{3}$ QLEDs via surface ligand density control, Adv. Mater. 29 (2017) 1603885.

[35] A.V. Antanovich, A.V. Prudnikau, D. Melnikau, Y.P. Rakovich, A. Chuvilin, U. Woggon, A.W. Achtsteine, M.V. Artemyev, Colloidal synthesis and optica properties of type-II CdSe-CdTe and inverted CdTe-CdSe core-wing heteronanoplatelets, Nanoscale 7 (2015) 8084-8092.

[36] Y. Kelestemur, M. Olutas, S. Delikanli, B. Guzelturk, M.Z. Akgul, H.V. Demir, TypeII colloidal quantum wells: CdSe/CdTe core/crown heteronanoplatelets, J. Phys. Chem. C 119 (2015) 2177-2185.

[37] Y. Shirasaki, G.J. Supran, M.G. Bawendi, V. Bulovic, Emergence of colloidal quantum-dot light-emitting technologies, Nat. Photonics 7 (2013) 13-23.

[38] B. Liu, L. Wang, D.Y. Gao, J.H. Zou, H.L. Ning, J.B. Peng, Y. Cao, Extremely high efficiency and ultrasimplified hybrid white organic light-emitting diodes exploiting double multifunctional blue emitting layers, Light: Sci. Appl. 5 (2016) e16137.

[39] Q. Li, B. Zhou, J.R. McBride, T. Lian, Efficient diffusive transport of hot and cold excitons in colloidal type II CdSe/CdTe core/crown nanoplatelet heterostructures, ACS Energy Lett. 2 (2017) 174-181.

[40] H. Mu, W. Li, R. Jones, A. Steckl, D. Klotzkin, A comparative study of electrode effects on the electrical and luminescent characteristics of Alq $/$ /TPD OLED: improvements due to conductive polymer (PEDOT) anode, J. Lumin. 126 (2007) 225-229.

[41] Y. Zhang, J. Yu, J. Huang, R. Jiang, G. Huang, Fill factor of planar heterojunction organic solar cells with varied donor materials, J. Phys. D: Appl. Phys. 45 (2012) 175101.

[42] B. Liu, M. Xu, L. Wang, X. Yan, H. Tao, Y. Su, D. Gao, L. Lan, J. Zou, J. Peng, Investigation and optimization of each organic layer: a simple but effective approach towards achieving high-efficiency hybrid white organic light-emitting diodes, Org. Electron. 15 (2014) 926-936.

[43] Q. Wang, D. Ma, Management of charges and excitons for high-performance white organic light-emitting diodes, Chem. Soc. Rev. 39 (2010) 2387-2398.

[44] B. Liu, M. Xu, L. Wang, H. Tao, Y. Su, D. Gao, J. Zou, L. Lan, J. Peng, Comprehensive study on the electron transport layer in blue fluorescent organic light-emitting diodes, ECS J. Solid State Sci. Technol. 2 (2013) R258-R261.

[45] W. Ji, S. Liu, H. Zhang, R. Wang, W. Xie, H. Zhang, Ultrasonic spray processed, highly efficient all-inorganic quantum-dot light-emitting diodes, ACS Photonics 4 (2017) 1271-1278.

[46] B. Liu, H. Nie, X. Zhou, S. Hu, D. Luo, D. Gao, J. Zou, M. Xu, L. Wang, Z. Zhao, A. Qin, J. Peng, H. Ning, Y. Cao, B.Z. Tang, Manipulation of charge and exciton distribution based on blue aggregation-induced emission fluorophors: a novel concept to achieve high-performance hybrid white organic light-emitting Diodes, Adv. Funct. Mater. 26 (2016) 776-783.

[47] B. Liu, L. Wang, J. Zou, H. Tao, Y. Su, D. Gao, M. Xu, L. Lan, J. Peng, Investigation on spacers and structures: a simple but effective approach toward high-performance hybrid white organic light emitting diodes, Synth. Met. 184 (2013) 5-9.

[48] Y. Sun, N. Giebink, H. Kanno, B. Ma, M. Thompson, S.R. Forrest, Management of singlet and triplet excitons for efficient white organic light-emitting devices, Nature 440 (2006) 908-912.

[49] S. Reineke, F. Lindner, G. Schwartz, N. Seidler, K. Walzer, B. Lussem, K. Leo, White organic light-emitting diodes with fluorescent tube efficiency, Nature 459 (2009) 234-239.

[50] N. Sun, Q. Wang, Y. Zhao, Y. Chen, D. Yang, F. Zhao, J. Chen, D. Ma, High-performance hybrid white organic light-emitting devices without interlayer between fluorescent and phosphorescent emissive regions, Adv. Mater. 26 (2014) 1617-1621.

[51] W. Ji, P. Jing, L. Zhang, D. Li, Q. Zeng, S. Qu, J. Zhao, The work mechanism and sub-bandgap-voltage electroluminescence in inverted quantum dot light-emitting diodes, Sci. Rep. 4 (2014) 6974. 
[52] T. Matsushima, G. Jin, Y. Kanai, T. Yokota, S. Kitada, T. Kishi, H. Murata, Formation of ohmic carrier injection at anode/organic interfaces and carrier transport mechanisms of organic thin films, Mater. Res. Soc. Symp. Proc. 1154 (2009) 1154 B10-92.

[53] K.R. Choudhury, J. Lee, N. Chopra, A. Gupta, X. Jiang, F. Amy, F. So, Highly efficient hole injection using polymeric anode materials for small-molecule organic light-emitting diodes, Adv. Funct. Mater. 19 (2009) 491-496.

[54] J.H. Kwak, W.K. Bae, D.G. Lee, I. Park, J.H. Lim, M.J. Park, H.D. Cho, H.J. Woo, D.Y. Yoon, K.H. Char, S.H. Lee, C.H. Lee, Bright and efficient full-color colloidal quantum dot light emitting diodes using an inverted device structure, Nano Lett. 12 (2012) 2362-2366.

[55] M.D. Ho, D. Kim, N. Kim, S.M. Cho, H. Chae, Polymer and small molecule mixture for organic hole transport layers in quantum dot light-emitting diodes, ACS Appl. Mater. Interfaces 5 (2013) 12369-12374.

[56] W. Ji, Y. Lv, P. Jing, H. Zhang, J. Wang, H. Zhang, J. Zhao, Highly efficient and low turn-on voltage quantum dot light-emitting diodes by using a stepwise hole-transport layer, ACS Appl. Mater. Interfaces 7 (2015) 15955-15960.

[57] J. Kwak, W.K. Bae, D. Lee, I. Park, J. Lim, M. Park, H. Cho, H. Woo, D.Y. Yoon, K. Char, S. Lee, C. Lee, Bright and efficient full-color colloidal quantum dot lightemitting diodes using an inverted device structure, Nano Lett. 12 (2012) 2362-2366.

[58] W.K. Bae, Y. -S. Park, J. Lim, D. Lee, L.A. Padilha, H. McDaniel, I. Robel, C. Lee, J.M. Pietyrga, V.I. Klimov, Controlling the influence of auger recombination on the performance of quantum-dot light-emitting diodes, Nat. Commun. 4 (2013) 2661.

[59] X. Yang, E. Mutlugun, C. Dang, K. Dev, Y. Gao, S.T. Tan, X.W. Sun, H.V. Demir, Highly flexible, electrically driven, top-emitting, quantum dot light-emitting stickers, ACS Nano 8 (2014) 8224-8231.

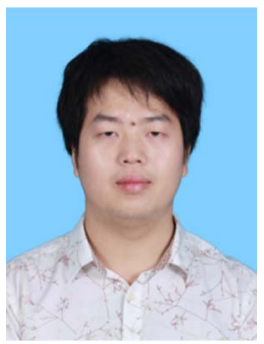

Baiquan Liu received the B.S. degree in Electronics Science and Technology from Shaanxi University of Science and Technology in 2011, Ph.D. degree from Materials Physics and Chemistry at South China University of Technology in 2016. Now, he is working at Nanyang Technological University. His current scientific interest is focused on the physics and chemistry of organic, nanoplatelet, quantumdot and perovskite light-emitting diodes.

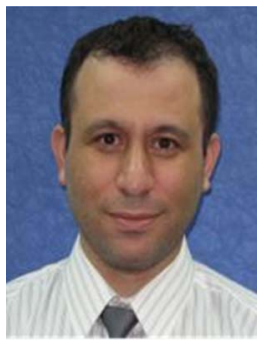

Savas Delikanli received his Ph.D. degree in Physics from State University of New York at Buffalo in 2011. He continued his research as a Post-Doctoral researcher at SUNY at Buffalo until 2013. Then, he worked two years as a PostDoctoral researcher at National Nanotechnology Research Center (UNAM) at Bilkent University. Dr. Delikanli has been working as a Post-Doctoral researcher at Nanyang Technological University since 2016 . His research mainly focuses on the synthesis of colloidal quantum dots, nanoplatelets and magnetic nanoparticles, and optical, magnetic, and magneto-optical characterization of nanostructures.

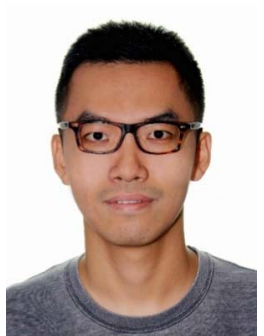

Yuan Gao completed his undergraduate study in Physics at Shandong University, China. He did his Ph.D. between Aug. 2011 to Apr. 2016 at Division of Physics and Applied Physics, Nanyang Technological University (NTU), Singapore under the supervision of Prof. Hilmi Volkan Demir and Prof. Handong Sun. From May 2016 to Sept. 2017, he worked at School of Electrical and Electronic Engineering, NTU Singapore as a research fellow. Currently, he is working at the University of Toronto as a Post-Doctoral Fellow. His main research interests are colloidal quantum dot synthesis, lasing and the laser spectroscopic study.

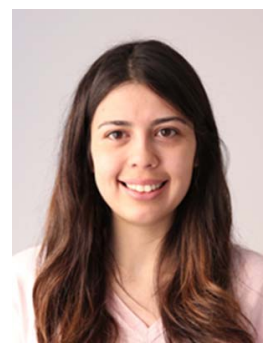

Didem Dede is a M.Sc. student working under the supervision of Prof. Hilmi Volkan Demir in Materials Science and Nanotechnology Program at Bilkent University. Her research focuses on the colloidal synthesis of semiconductor nanocrystals having various architectures especially nanoplatelets for the achievement of high performance required light-harvesting and emitting applications.

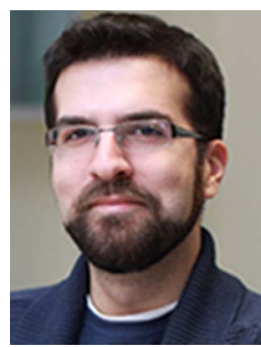

Kivanc Gungor received his B.Sc. (2010) and M.Sc. (2013) degrees in the Department of Physics and Department of Electrical and Electronics Engineering at Bilkent University, respectively. He is currently pursuing his Ph.D. under guidance of Prof. Hilmi Volkan Demir in the Department of Electrical and Electronics Engineering. His current research involves establishing possible application platforms for semiconductor nanocrystals. Specifically, he focuses on developing luminescent solar concentrators using novel nanocrystals and plasmonic polarizing surfaces for display applications.

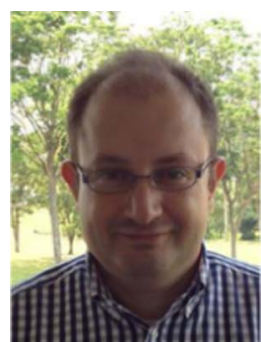

Hilmi Volkan Demir received his M.S. and Ph.D. degree in electrical engineering from Stanford University, in 2000 and 2004, respectively. In 2004, he joined Bilkent University as a faculty member and is a Professor at the Department of Electrical-Electronics Engineering and the Department of Physics. $\mathrm{He}$ is concurrently Nanyang Professor at the School of Electrical Electronic Engineering and, Physical and Mathematical Sciences, NTU, and the Director of the LUMINOUS! Center of Excellence for Semiconductor Lighting and Displays. His current research interests include energy-saving LEDs for quality lighting, the science of excitonics for high-efficiency light harvesting, and nanocrystal optoelectronics. 